\title{
Killing quantum entanglement by acceleration or a black hole
}

\author{
Yue Dai, ${ }^{a}$ Zhejun Shen ${ }^{a}$ and Yu Shi ${ }^{a, b, 1}$ \\ ${ }^{a}$ Center for Field Theory and Particle Physics, \\ Department of Physics \& State Key Laboratory of Surface Physics, Fudan University, \\ Shanghai 200433, China \\ ${ }^{b}$ Collaborative Innovation Center of Advanced Microstructures, Fudan University, \\ Shanghai 200433, China \\ E-mail: dy1983@gmail.com, victorshenzj@gmail.com, yushi@fudan.edu.cn
}

\begin{abstract}
We consider two entangled accelerating qubits coupled with real scalar fields, each described by the Unruh-Wald model. It is demonstrated that because of the Unruh effect of the fields, the bipartite entanglement between the two qubits suddenly dies when the acceleration of one or more qubits are large enough. We also consider three entangled accelerating qubits in GHZ state and in W state, with equal acceleration-frequency ratio, and found that in either state, the tripartite entanglement suddenly dies at a certain value of acceleration-frequency ratio. The equivalence between the Rindler metric and the Schwarzschild metric in the vicinity of the horizon of a black hole implies that for two entangled qubits outside a black hole, the entanglement suddenly dies when one or both of the qubits are close enough to the horizon, while the three entangled qubits in GHZ or W state, the tripartite entanglement suddenly dies when these qubits are close enough to the horizon.
\end{abstract}

KeYwords: Models of Quantum Gravity, Black Holes, Quantum Dissipative Systems

ArXIV EPRINT: 1507.00612

\footnotetext{
${ }^{1}$ Corresponding author.
} 


\section{Contents}

1 Introduction 1

2 Formalism 2

3 Entangled states of the two detecting qubits 4

4 von Neumann entropy $S\left(\rho_{A B}\right)$ and mutual information $I(A: B) \quad 5$

$\begin{array}{lll}5 & \text { Entanglement between qubits } A \text { and } B & 7\end{array}$

$\begin{array}{llll}6 & \text { Tripartite entanglement in three entangled accelerating qubits } & 8\end{array}$

$\begin{array}{lll}7 & \text { Discussion and summary } & 11\end{array}$

\section{Introduction}

Recent two decades witnessed both intensive and extensive investigations on quantum entanglement, "the characteristic trait of quantum mechanics" in the words of Schrödinger [1]. More recently, this trend has been extended to the realms of high energy physics. The relativistic effects of acceleration and gravitation on quantum entanglement were investigated, shedding new light on the subject of quantum effects of gravity.

A particularly concerned subject is the Unruh effect, that is, the particle content of a field is observer-dependent, and thus an accelerating detector in the Minkowski vacuum of a field feels a thermal bath of particles of this field [2-8]. The consequences of the Unruh effect on various kinds of entanglement have been studied. For example, because of the Unruh effect, a state of two field modes that is maximally entangled in an inertial frame becomes less entangled when one of the modes is observed by an accelerating detector, and degrades with the increase of acceleration, towards zero at the limit of infinite acceleration [9]. Analogous entanglement degradation occurs for field modes observed by two detectors close to the horizon of a black hole, with one of them freely falling while the other barely escapes $[9,10]$. This problem was then extended to the case of three entangled modes, and it was found that when one of the modes is observed by an accelerating detector, the tripartite entanglement, which cannot be reduced to all kinds of bipartite entanglement, does not approach zero in the infinite acceleration limit $[11,12]$. Extension of such studies to Fermion fields was also made [13-16]. These results are consistent with the general feature that entanglement of a field state depends on the choice of single particle modes [17]. Entanglement between detectors have also been studied in the case that one of the two entangled detectors accelerates while the other moves uniformly, and was found to exhibit entanglement sudden death [18]. One approach is to model the detectors as harmonic 
oscillators [19], for which calculations were also made on the physical details [20, 21]. Another approach is to model the detectors as qubits [22]. Yet another approach is to consider the detectors as an open quantum system [23-25]. Other quantum informational quantities such as discord [26, 27] and quantum Fisher information [28, 29] have also been studied. So far there was no work on the case that more than one of the entangled detectors accelerate.

In this paper, we consider two causally separated but quantum-entangled qubits, each of which independently accelerates and is coupled with a scalar field as described by the Unruh-Wald model. Our work originated in generalizing a previous work on the decoherence of one qubit due to acceleration [30]. We show that because of the Unruh effect of the fields, the entanglement between the qubits vanishes at finite values of acceleration instead of in the infinite limit, i.e. exhibit entanglement sudden death. Our result implies that outside of the black hole, the entanglement between the two entangled detecting qubits vanishes when one or both of the qubits are close enough to the horizon. We also report a result on the tripartite entanglement in three entangled accelerating qubits.

\section{Formalism}

Let us consider two qubits $A$ and $B$ far away from each other, that is, we assume there is no causal connection between the two qubits. For each qubit, we apply the model of the Unruh and Wald [6].

The Hamiltionian of each qubit $q(q=A, B)$ is

$$
H_{q}=\Omega_{q} Q_{q}^{\dagger} Q_{q}
$$

where the creation operator $Q_{q}^{\dagger}$ and annihilation operator $Q_{q}$ are defined by $Q_{q}|0\rangle_{q}=$ $Q_{q}^{\dagger}|1\rangle_{q}=0, Q_{q}^{\dagger}|0\rangle_{q}=|1\rangle_{q}$ and $Q_{q}|1\rangle_{q}=|0\rangle_{q}$, with subscript $q=a, b . \Omega_{q}$ gives the energy difference between eigenstates $|1\rangle_{q}$ and $|0\rangle_{q}$.

Each qubit is locally coupled with a field $\Phi_{q}$ within a small region around it, the interaction Hamiltonian being

$$
H_{I_{q}}\left(t_{q}\right)=\epsilon_{q}\left(t_{q}\right) \int_{\Sigma_{q}} \Phi_{q}(\mathbf{x})\left[\psi_{q}(\mathbf{x}) Q_{q}+\psi_{q}^{*}(\mathbf{x}) Q_{q}^{\dagger}\right] \sqrt{-g} d^{3} x
$$

where $\mathbf{x}$ and $t_{q}$ are spacetime coordinates in the comoving frame of the qubit, the integral is over the spacelike Cauchy surface $\Sigma_{q}$ at given time $t_{q}, \epsilon_{q}\left(t_{q}\right)$ is the coupling constant with a finite duration of qubit-field interaction, $\psi_{q}(\mathbf{x})$ is a smooth function nonvanishing within a small volume around the qubit [5]. The fields $\Phi_{A}$ and $\Phi_{B}$ could be the same or different.

We presume that the distance between the two qubits is so large that there is no physical coupling or influence between the neighboring fields of the two qubits during the interaction times. The total Hamiltonian is simply $H_{A}+H_{\Phi_{A}}+H_{I_{A}}+H_{B}+H_{\Phi_{B}}+H_{I_{B}}$, where $H_{\Phi_{q}}$ is the Klein-Gordon Hamiltonian for $\Phi_{q}$. In the Minkowski spacetime, each qubit is confined in its own Rindler wedge and possesses boost Killing fields which are timelike. The only extra constraint on the trajectories of the two qubits is that the time 
interval of the interaction between each qubit and its neighboring field multiplied by the speed of light is smaller than the shortest distance between the interaction regions of the two qubits.

Therefore after a time duration longer than the interacting times $T_{q} \gg 1 / \Omega_{q}$, the state of the whole system in the interaction picture is transformed by

$$
U_{A} \otimes U_{B}
$$

where $U_{q}$ is the unitary transformation acting on qubit $q$ and the field $\Phi_{q}$ in its neighboring region, as given by the Unruh-Wald model. To the first order [6],

$$
U_{q} \approx 1-i \int \Phi_{q 0}\left(t^{\prime}, \mathbf{x}\right) \epsilon_{q}\left(t^{\prime}\right)\left[Q_{q 0} e^{-i \Omega_{q} t^{\prime}} \psi_{q}(\mathbf{x})+Q_{q 0}^{\dagger} e^{i \Omega_{q} t^{\prime}} \psi_{q}^{*}(\mathbf{x})\right] \sqrt{-g^{\prime}} d^{3} x d t^{\prime},
$$

where $\Phi_{q 0}$ and $Q_{q 0}$ are $\Phi_{q}$ and $Q_{q}$ for $\epsilon_{q}=0$, i.e. when the qubit-field coupling is turned off. It can be obtained that

$$
U_{q} \approx 1+i Q_{q 0} a^{\dagger}\left(\Gamma_{q}^{*}\right)-i Q_{q 0}^{\dagger} a\left(\Gamma_{q}^{*}\right)
$$

where $a\left(\Gamma_{q}^{*}\right)$ and $a^{\dagger}\left(\Gamma_{q}^{*}\right)$ are the annihilation and the creation operators of $\Gamma_{q}^{*}$, with

$$
\Gamma_{q}(x) \equiv-2 i \int\left[G_{R}\left(x ; x^{\prime}\right)-G_{A}\left(x ; x^{\prime}\right)\right] \epsilon_{q}\left(t^{\prime}\right) e^{i \Omega t} \psi_{q}^{*}\left(\mathbf{x}^{\prime}\right) \sqrt{-g^{\prime}} d^{4} x^{\prime},
$$

$G_{R q}$ and $G_{A q}$ being the retarded and advanced Green functions of the field $\Phi_{q}$, respectively.

For any mode $\chi_{q}$, we can write

$$
\begin{aligned}
a\left(\Gamma_{q}^{*}\right) & =\left\langle\Gamma_{q}^{*}, \chi_{q}\right\rangle a\left(\chi_{q}\right)+\left\langle\Gamma_{q}^{*}, \chi_{q}^{\prime}\right\rangle a\left(\chi_{q}^{\prime}\right), \\
a^{\dagger}\left(\Gamma_{q}^{*}\right) & =\left\langle\Gamma_{q}^{*}, \chi_{q}\right\rangle^{*} a^{\dagger}\left(\chi_{q}\right)+\left\langle\Gamma_{q}^{*}, \chi_{q}^{\prime}\right\rangle^{*} a^{\dagger}\left(\chi_{q}^{\prime}\right)
\end{aligned}
$$

where $\chi_{q}^{\prime}$ is some mode orthogonal to $\chi_{q} \cdot\left\langle\Gamma_{q}^{*}, \chi_{q}\right\rangle=\frac{i}{2} \int_{\Sigma_{q}}\left[\Gamma_{q} \partial_{\mu} \chi_{q}-\left(\partial_{\mu} \Gamma_{q}\right) \chi_{q}\right] d S^{\mu}$, where $\Sigma_{q}$ is some Cauchy surface. This inner product can be assumed to be negligible unless $\chi_{q}$ is at a frequency $\approx \Omega_{q}$. Therefore, each qubit $q$ is only coupled with the field mode $\chi\left(\Omega_{q}\right)$ with frequency $\Omega_{q}$, which is further assumed to be nondegenerate. Hence we only need to consider $\chi_{\Omega_{A}}$ and $\chi_{\Omega_{B}}$ in studying the qubits $A$ and $B$. All the other modes are decoupled with the qubits.

Now we consider the Fock state $|n\rangle_{\Omega_{q}}$, containing $n$ particles in the mode $\chi\left(\Omega_{q}\right)$ of the field $\Phi_{q}$, as observed in the Rindler wedge confining qubit $q$,

$$
\begin{aligned}
a\left(\Gamma_{q}^{*}\right)|n\rangle_{\chi_{q}} & =\mu_{q} a\left(\Omega_{q}\right)|n\rangle_{\Omega_{q}}=\mu_{q} \sqrt{n}|n-1\rangle_{\Omega_{q}}, \\
a^{\dagger}\left(\Gamma_{q}^{*}\right)|n\rangle_{\Omega_{q}} & =\mu_{q}^{*} a^{\dagger}\left(\Omega_{q}\right)|n\rangle_{\Omega_{q}}=\mu_{q}^{*} \sqrt{n+1}|n+1\rangle_{\Omega_{q}},
\end{aligned}
$$

where $\mu_{q} \equiv\left\langle\Gamma_{q}^{*}, \chi_{\Omega_{q}}\right\rangle=\int \epsilon_{q}(t) e^{i \Omega_{q} t} \psi_{q}^{*}(\mathbf{x}) \chi_{\Omega_{q}}(t, \mathbf{x}) \sqrt{-g} d^{4} x$.

Hence $U_{q}$ evolves only the qubit $q$ and the mode $\chi_{\Omega_{q}}$, while the other modes of $\Phi_{q}$ are not affected and can be ignored,

$$
\begin{aligned}
& U_{\Omega_{q}}|0\rangle_{q}|n\rangle_{\Omega_{q}}=|0\rangle_{q}|n\rangle_{\Omega_{q}}-i \sqrt{n} \mu_{q}|1\rangle_{q}|n-1\rangle_{\Omega_{q}}, \\
& U_{\Omega_{q}}|1\rangle_{q}|n\rangle_{\Omega_{q}}=|1\rangle_{q}|n\rangle_{\Omega_{q}}+i \sqrt{n+1} \mu_{q}^{*}|0\rangle_{q}|n+1\rangle_{\Omega_{q}} .
\end{aligned}
$$




\section{Entangled states of the two detecting qubits}

We now suppose the initial state of the two qubits to be

$$
\left|\Psi_{i}\right\rangle=\alpha|0\rangle_{A}|1\rangle_{B}+\beta|1\rangle_{A}|0\rangle_{B},
$$

where $\alpha$ and $\beta$ are superposition coefficients satisfying $|\alpha|^{2}+|\beta|^{2}=1$. The results for the initial state of the form of $\alpha|0\rangle_{A}|0\rangle_{B}+\beta|1\rangle_{A}|1\rangle_{B}$ are similar. Without causal connection between the two qubits or between the fields, each qubit detects a thermal bath of the Unruh particles determined by its own acceleration. With each qubit in its own Rindler wedge, the initial state of the whole system, as observed by the observers comoving with the qubits, is described by the density matrix

$$
\rho_{i}=\left|\Psi_{i}\right\rangle\left\langle\Psi_{i}\right| \otimes \rho_{\Omega_{A}} \otimes \rho_{\Omega_{B}} \otimes \rho^{\prime},
$$

where

$$
\rho_{\Omega_{q}}=C_{q} \sum_{n_{q}} e^{-2 \pi n_{q} \Omega_{q} / a_{q}}\left|n_{q}\right\rangle_{\Omega_{q}}\left\langle n_{q}\right|,
$$

$a_{q}$ is the acceleration of qubit $q, C_{q} \equiv \sqrt{1-e^{-2 \pi \Omega_{q} / a_{q}}}, \rho^{\prime}$ is the state of the other decoupled modes and is ignored henceforth.

The final state of the system in the interaction picture is

$$
\rho_{f}=U_{B}^{\dagger} U_{A}^{\dagger} \rho_{i} U_{A} U_{B}
$$

which can be evaluated by substituting eqs. (2.9) and (2.10). Subsequently by tracing out the fields, we obtain the reduced density matrix of the two qubits, with respect to the comoving observers

$$
\begin{aligned}
\rho_{A B}= & C_{A}^{2} C_{B}^{2} \sum_{n_{A}, n_{B}} \frac{e^{-2 \pi\left(n_{A} \Omega_{A} / a_{A}+n_{B} \Omega_{B} / a_{B}\right)}}{Z_{n_{A} n_{B}}} \\
\times & \left\{\left[|\alpha|^{2}\left(n_{B}+1\right)\left|\mu_{B}\right|^{2}+|\beta|^{2}\left(n_{A}+1\right)\left|\mu_{A}\right|^{2}\right]|00\rangle\langle 00|\right. \\
& +\alpha \beta^{*}|01\rangle\left\langle 10\left|+\beta \alpha^{*}\right| 10\right\rangle\langle 01| \\
& +\left[|\alpha|^{2}+|\beta|^{2} n_{B}\left(n_{A}+1\right)\left|\mu_{B}\right|^{2}\left|\mu_{A}\right|^{2}\right]|01\rangle\langle 01| \\
& +\left[|\alpha|^{2} n_{A}\left(n_{B}+1\right)\left|\mu_{A}\right|^{2}\left|\mu_{B}\right|^{2}+|\beta|^{2}\right]|10\rangle\langle 10| \\
& \left.+\left[|\alpha|^{2} n_{A}\left|\mu_{A}\right|^{2}+|\beta|^{2} n_{B}\left|\mu_{B}\right|^{2}\right]|11\rangle\langle 11|\right\},
\end{aligned}
$$

where $Z_{n_{A} n_{B}} \equiv 1+n_{A}\left|\mu_{A}\right|^{2}+n_{B}\left|\mu_{B}\right|^{2}+n_{A} n_{B}\left|\mu_{A}\right|^{2}\left|\mu_{B}\right|^{2}+|\alpha|^{2}\left(\left|\mu_{B}\right|^{2}+n_{A}\left|\mu_{A}\right|^{2}\left|\mu_{B}\right|^{2}\right)+$ $|\beta|^{2}\left(\left|\mu_{A}\right|^{2}+n_{B}\left|\mu_{B}\right|^{2}\left|\mu_{A}\right|^{2}\right)$.

In the case that qubit $A$ moves uniformly while qubit $B$ accelerates, $\rho_{A B}$ is

$$
\begin{aligned}
\rho_{A B}= & C_{B}^{2} \sum_{n_{B}} \frac{e^{-2 \pi n_{B} \Omega_{B} / a_{B}}}{Z_{n_{B}}} \\
\times & \left\{\left[|\alpha|^{2}\left(n_{B}+1\right)\left|\mu_{B}\right|^{2}+|\beta|^{2}\left|\mu_{A}\right|^{2}\right]|00\rangle\langle 00|\right. \\
& +\alpha \beta^{*}|01\rangle\left\langle 10\left|+\beta \alpha^{*}\right| 10\right\rangle\langle 01| \\
& +\left[|\alpha|^{2}+|\beta|^{2} n_{B}\left|\mu_{B}\right|^{2}\left|\mu_{A}\right|^{2}\right]|01\rangle\langle 01| \\
& \left.+|\beta|^{2}|10\rangle\left\langle\left. 10|+| \beta\right|^{2} n_{B}\left|\mu_{B}\right|^{2} \mid 11\right\rangle\langle 11|\right\},
\end{aligned}
$$

where $Z_{n_{B}} \equiv 1+n_{B}\left|\mu_{B}\right|^{2}+|\alpha|^{2}\left|\mu_{B}\right|^{2}+|\beta|^{2}\left|\mu_{A}\right|^{2}+|\beta|^{2} n_{B}\left|\mu_{A}\right|^{2}\left|\mu_{B}\right|^{2}$. 


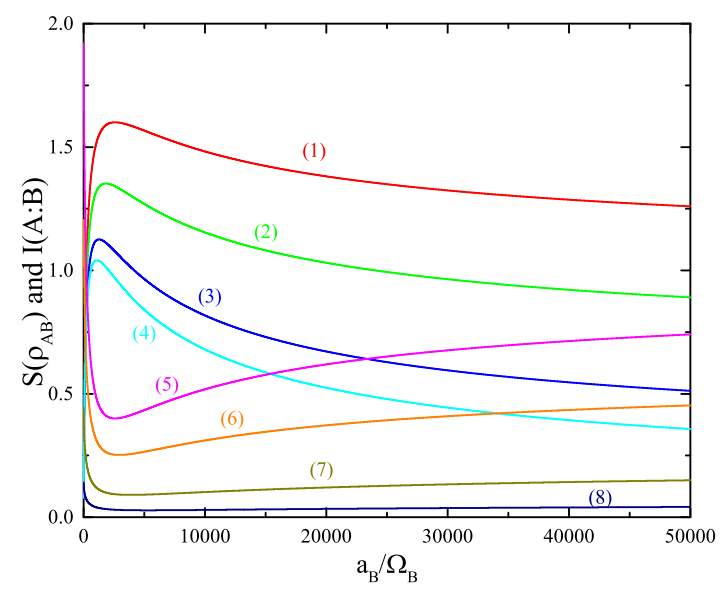

Figure 1. $S\left(\rho_{A B}\right)$ [plots (1) to (4)] and mutual information $I(A: B)$ [plots (5) to (8)] as functions of the acceleration-frequency ratio of qubit $B$, in the case that qubit $A$ moves uniformly, for different initial states. $(1,5): \alpha=1 / \sqrt{2},(2,6): \alpha=0.4,(3,7): \alpha=0.2,(4,8): \alpha=0.1$.

We now study the correlation and entanglement in $\rho_{A B}$. Note that the entanglement and correlation are respectively the same in Schrödinger and interaction pictures.

\section{4 von Neumann entropy $S\left(\rho_{A B}\right)$ and mutual information $I(A: B)$}

The von Neumann entropy

$$
S\left(\rho_{A B}\right) \equiv-\operatorname{Tr} \rho_{A B} \log \rho_{A B}
$$

is a measure of mixture of $\rho_{A B}$. On the other hand, in the Minkowski frame, the state of the whole system is a pure state, and $S\left(\rho_{A B}\right)$ quantifies the entanglement between the two qubits on one hand, and the fields on the other.

From $S\left(\rho_{A B}\right)$, we also calculate the mutual information

$$
I(A: B) \equiv S\left(\rho_{A}\right)+S\left(\rho_{B}\right)-S\left(\rho_{A B}\right),
$$

where

$$
\begin{aligned}
& \rho_{A}=\operatorname{Tr}_{B} \rho_{A B}, \\
& \rho_{B}=\operatorname{Tr}_{A} \rho_{A B} .
\end{aligned}
$$

$I(A: B)$ is the difference between the sum of the entropies of $A$ and $B$ as a whole on one hand, and the entropy of $A$ plus $B$ as a whole on the other, and is thus a quantification of the total correlation contributed by both entanglement and classical correlation. In the numerical calculations throughout this paper, the bases of the logarithms are chosen to be 2 , and the parameters $\mu_{A}, \mu_{B}$ are both set to be 0.1 .

$S\left(\rho_{A B}\right)$ and $I(A: B)$ are depicted together in figures 1 to 3 for three cases. The result for the case of qubit $A$ uniformly moving while qubit $B$ accelerating is shown in figure 1. 


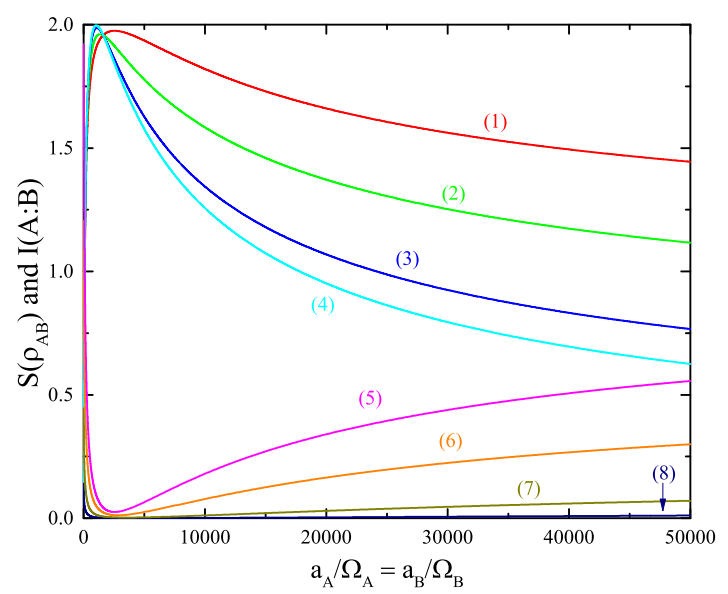

Figure 2. $S\left(\rho_{A B}\right)$ [plots (1) to (4)] and mutual information $I(A: B)$ [plots (5) to (8)] as functions of the acceleration-frequency ratio, which is assumed to be the same for the two qubits, for different initial states. $(1,5): \alpha=1 / \sqrt{2},(2,6): \alpha=0.4,(3,7): \alpha=0.2,(4,8): \alpha=0.1$. The extreme values are insensitive to the initial state.

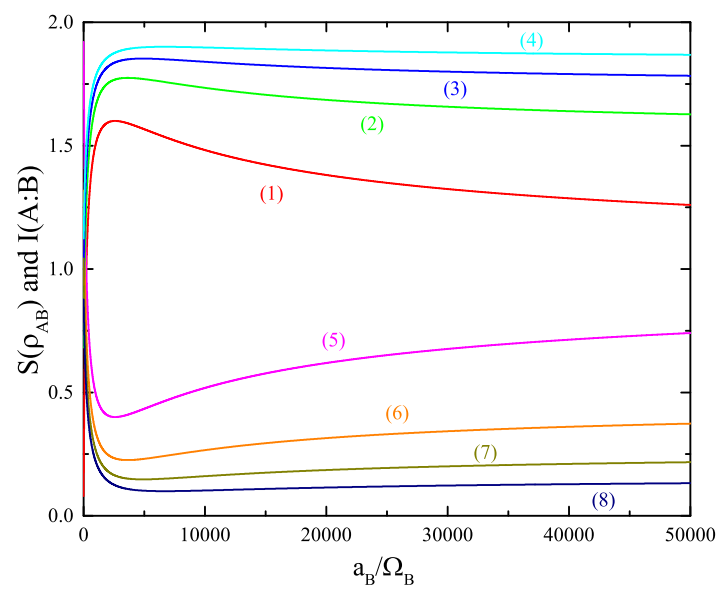

Figure 3. $S\left(\rho_{A B}\right)$ [plots (1) to (4)] and mutual information $I(A: B)$ [plots (5) to (8)] as functions of the acceleration-frequency ratio of qubit $B$, for different given values of acceleration-frequency ratio of qubit $A:(1,5): a_{A} / \Omega_{A}=0,(2,6): a_{A} / \Omega_{A}=100,(3,7): a_{A} / \Omega_{A}=200,(4,8): a_{A} / \Omega_{A}=300$. It is set that $\alpha=\beta=\frac{1}{\sqrt{2}}$.

The special case of two maximally entangled qubits with one of them uniformly moving was previously studied by using a different approach and assuming no coupling between the uniformly moving qubit and the field [7]. In our studies, both qubits always couple with the fields. Figure 2 depicts the result for the case that the acceleration-frequency ratios of the two qubits are always equal. Figure 3 gives the results for various given values of $a_{A} / \Omega_{A}$. As shown in these figures, when both $a_{A} / \Omega_{A}$ and $a_{B} / \Omega_{B}$ are close to $0, \rho_{A B}$ is close to the pure state $\left|\Psi_{i}\right\rangle$, hence $\rho_{A}$ is close to $|\alpha|^{2}|0\rangle\left\langle\left. 0|+| \beta\right|^{2} \mid 1\right\rangle\langle 1|$ while $\rho_{B}$ is close to $|\beta|^{2}|0\rangle\left\langle\left. 0|+| \alpha\right|^{2} \mid 1\right\rangle\langle 1|$, therefore $S\left(\rho_{A B}\right)$ is close to 0 while $I(A: B)$ is close to $-2|\alpha|^{2} \log |\alpha|^{2}-2|\beta|^{2} \log |\beta|^{2}$. With the increase of one or both of the acceleration-frequency ratios, $S\left(\rho_{A B}\right)$ quickly increases up 


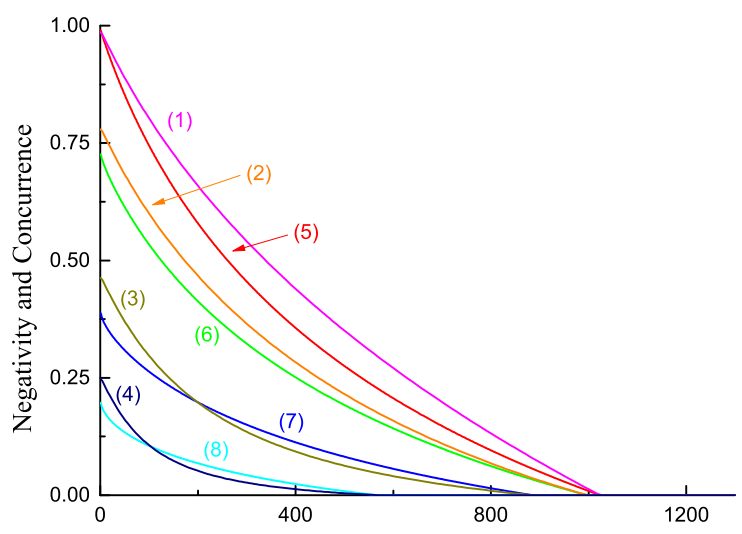

Figure 4. Logarithmic negativity [plots (1) to (4)] and concurrence [plots (5) to (8)] as functions of the acceleration-frequency ratio of qubit $B$, in the case that qubit $A$ moves uniformly, for different initial state. $(1,5): \alpha=1 / \sqrt{2},(2,6): \alpha=0.4,(3,7): \alpha=0.2,(4,8): \alpha=0.1$.

to a maximum while $I(A: B)$ quickly decreases down to a minimum. The actual extreme values depend on the details of the dynamics, but we can make the following estimation. If $\rho_{A B}$ is maximally mixed, $S\left(\rho_{A B}\right)$ reaches the absolute maximum 2 while $S\left(\rho_{A}\right)$ and $S\left(\rho_{B}\right)$ reach the absolute maximum 1, because $\rho_{A B}$ is 4-dimensional while $\rho_{A}$ and $\rho_{B}$ are 2-dimensional. Therefore the minimal value of $I(A: B)$ is near 0 . When the accelerationfrequencies further increase, $S\left(\rho_{A B}\right)$ slowly decreases while $I(A: B)$ slowly increases. In the limit of $a_{B} / \Omega_{B} \rightarrow \infty$ while $a_{A} / \Omega_{A}=0, \rho_{A B} \rightarrow|\alpha|^{2}|00\rangle\left\langle\left. 00|+| \beta\right|^{2} \mid 11\right\rangle\langle 11|$. In the limit of both $a_{A} / \Omega_{A}$ and $a_{B} / \Omega_{B}$ approach $\infty, \rho_{A B} \rightarrow|\alpha|^{2}|10\rangle\left\langle\left. 10|+| \beta\right|^{2} \mid 01\right\rangle\langle 01|$. In both of these two limits, $S\left(\rho_{A B}\right), S\left(\rho_{A}\right), S\left(\rho_{B}\right)$ and $I(A: B)$ all approach $-|\alpha|^{2} \log |\alpha|^{2}-|\beta|^{2} \log |\beta|^{2}$.

\section{Entanglement between qubits $A$ and $B$}

Now we turn to the entanglement between qubits $A$ and $B$, which are in the mixed state $\rho_{A B}$. For the two-qubit mixed state $\rho_{A B}$, a measure of the entanglement is the logarithmic negativity

$$
\log \left\|\rho_{A B}^{T_{A}}\right\|,
$$

where $\left\|\rho_{A B}^{T_{A}}\right\|$ is the trace norm of $\rho_{A B}^{T_{A}}$, which is the partial transpose of $\rho_{A B}[31,32]$. Another entanglement measure is the concurrence

$$
C(\rho)=\max \left\{0, \lambda_{1}-\lambda_{2}-\lambda_{3}-\lambda_{4}\right\}
$$

where $\lambda_{i}(i=1,2,3,4)$ are decreasingly ordered eigenvalues of the matrix $\sqrt{\sqrt{\rho_{A B}} \tilde{A}_{A} B \sqrt{\rho_{A B}}}$, with $\rho_{A} B=\left(\sigma_{y} \otimes \sigma_{y}\right) \rho_{A B}^{*}\left(\sigma_{y} \otimes \sigma_{y}\right), \sigma_{y}$ being the $y$-component Pauli matrix [33].

As shown in figures 4 to 7 , the logarithmic negativity and concurrence decrease with the increase of the acceleration-frequency ratio of each qubit and, especially, suddenly dies at a finite value of the acceleration-frequency ratio. As can be seen in these figures, the acceleration-frequency ratio of one qubit at which the entanglement suddenly dies decreases with the increase of that of the other qubit. When one of them is zero, the other must be 


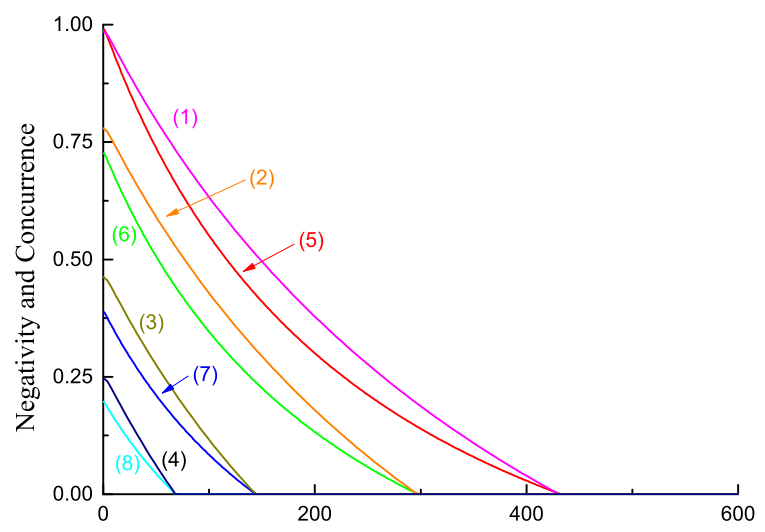

Figure 5. Logarithmic negativity [plots (1) to (4)] and concurrence [plots (5) to (8)] as functions of the acceleration-frequency ratios of $A$ and $B$, which are assumed to be the same, for different initial state. $(1,5): \alpha=1 / \sqrt{2},(2,6): \alpha=0.4,(3,7): \alpha=0.2,(4,8): \alpha=0.1$.

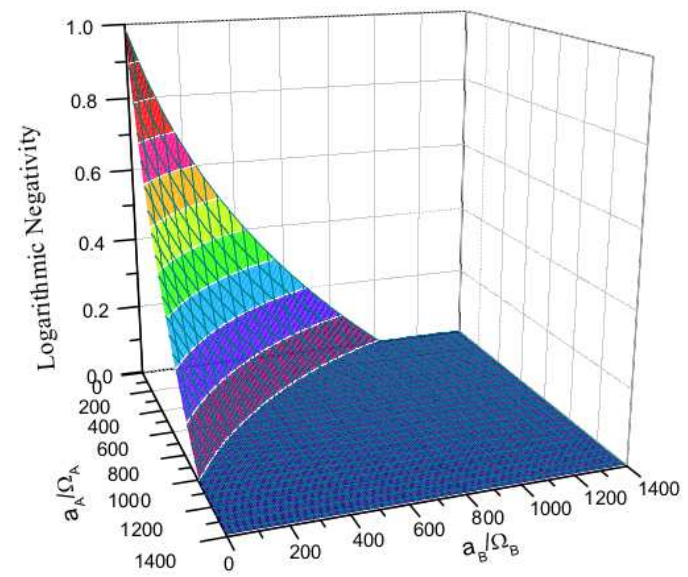

Figure 6. Logarithmic negativity as a function of $a_{A} / \Omega_{A}$ and $a_{B} / \Omega_{B} \cdot \alpha=\beta=\frac{1}{\sqrt{2}}$.

larger than some finite value. In figures 6 and 7, the 3D plots of logarithmic negativity and concurrence are symmetric with respective to the plane $a_{A} / \Omega_{A}=a_{B} / \Omega_{B}$, as can be seen in the the expression of $\rho_{A B}$. These $3 \mathrm{D}$ plots also indicate that entanglement sudden death occurs on a curve of $a_{A} / \Omega_{A}$ and $a_{B} / \Omega_{B}$.

\section{Tripartite entanglement in three entangled accelerating qubits}

We have also extended our study to three accelerating qubits $A, B$ and $C$, by using the formalism similar to the two-qubit case above. It is well known that there are two types of 3-qubit states, GHZ state

$$
|\mathrm{GHZ}\rangle_{A B C}=\frac{1}{\sqrt{2}}(|000\rangle+|111\rangle)
$$




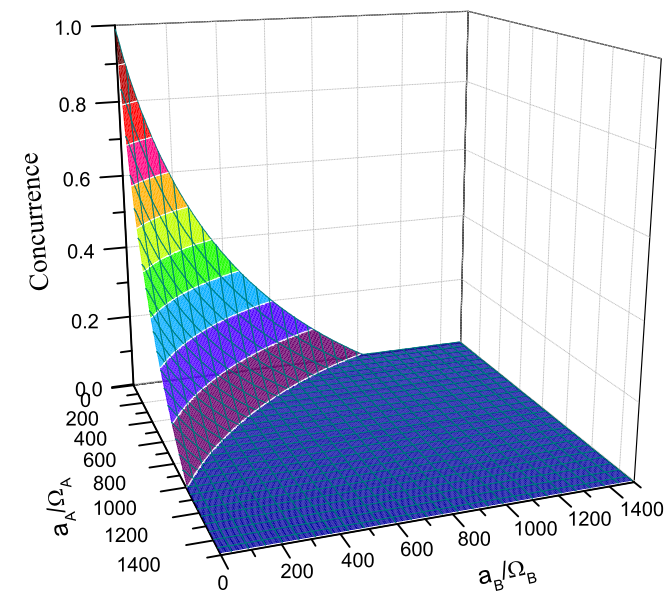

Figure 7. Concurrence as a function of the acceleration-frequency ratios of $A$ and $B . \alpha=\beta=\frac{1}{\sqrt{2}}$.

and $\mathrm{W}$ state

$$
|W\rangle_{A B C}=\frac{1}{\sqrt{3}}(|001\rangle+|010\rangle+|100\rangle),
$$

each representing a different type of tripartite entanglement [34].

We apply the above formalism to three entangled qubits $A, B$ and $C$, as described by eq. (2.1), each of which is locally coupled with a field $\Phi_{q}(q=A, B, C)$. For the reason given above, only the mode $\chi_{\Omega_{q}}$ needs to be considered. In this way, for GHZ state, we obtain the density matrix of the qubits,

$$
\begin{aligned}
\rho_{A B C}(\mathrm{GHZ})= & \frac{1}{2} C_{A}^{2} C_{B}^{2} C_{C}^{2} \sum_{n_{A}, n_{B}, n_{C}} \frac{e^{-2 \pi\left(n_{A} \Omega_{A} / a_{A}+n_{B} \Omega_{B} / a_{B}+n_{C} \Omega_{C} / a_{C}\right)}}{Z_{n_{A} n_{B} n_{C}}} . \\
& {\left[\left(1+\left(n_{A}+1\right)\left(n_{B}+1\right)\left(n_{C}+1\right)\left|\mu_{A}\right|^{2}\left|\mu_{B}\right|^{2}\left|\mu_{C}\right|^{2}\right)|000\rangle\langle 000|\right.} \\
& +\left(1+n_{A} n_{B} n_{C}\left|\mu_{A}\right|^{2}\left|\mu_{B}\right|^{2}\left|\mu_{C}\right|^{2}\right)|111\rangle\langle 111|+| 111\rangle\langle 000| \\
& +|000\rangle\left\langle 111\left|+\left(n_{A}\left|\mu_{A}\right|^{2}+\left(n_{B}+1\right)\left(n_{C}+1\right)\left|\mu_{B}\right|^{2}\left|\mu_{C}\right|^{2}\right)\right| 100\right\rangle\langle 100| \\
& +\left(n_{B}\left|\mu_{B}\right|^{2}+\left(n_{A}+1\right)\left(n_{C}+1\right)\left|\mu_{A}\right|^{2}\left|\mu_{C}\right|^{2}\right)|010\rangle\langle 010| \\
& +\left(n_{C}\left|\mu_{C}\right|^{2}+\left(n_{A}+1\right)\left(n_{B}+1\right)\left|\mu_{A}\right|^{2}\left|\mu_{B}\right|^{2}\right)|001\rangle\langle 001| \\
& +\left(n_{A} n_{B}\left|\mu_{A}\right|^{2}\left|\mu_{B}\right|^{2}+\left(n_{C}+1\right)\left|\mu_{C}\right|^{2}\right)|110\rangle\langle 110| \\
& +\left(n_{A} n_{C}\left|\mu_{A}\right|^{2}\left|\mu_{C}\right|^{2}+\left(n_{B}+1\right)\left|\mu_{B}\right|^{2}\right)|101\rangle\langle 101| \\
& \left.+\left(n_{B} n_{C}\left|\mu_{B}\right|^{2}\left|\mu_{C}\right|^{2}+\left(n_{A}+1\right)\left|\mu_{A}\right|^{2}\right)|011\rangle\langle 011|\right]
\end{aligned}
$$

where

$$
\begin{aligned}
Z_{n_{A} n_{B} n_{C}=} & 2+\left(2 n_{A}+1\right)\left|\mu_{A}\right|^{2}+\left(2 n_{B}+1\right)\left|\mu_{B}\right|^{2}+\left(2 n_{C}+1\right)\left|\mu_{C}\right|^{2} \\
& +\left(2 n_{A} n_{B}+n_{A}+n_{B}+1\right)\left|\mu_{A}\right|^{2}\left|\mu_{B}\right|^{2}+\left(2 n_{A} n_{C}+n_{A}+n_{C}+1\right)\left|\mu_{A}\right|^{2}\left|\mu_{C}\right|^{2} \\
& +\left(2 n_{B} n_{C}+n_{B}+n_{C}+1\right)\left|\mu_{B}\right|^{2}\left|\mu_{C}\right|^{2} \\
& +\left(2 n_{A} n_{B} n_{C}+n_{A} n_{B}+n_{B} n_{C}+n_{A} n_{C}+n_{A}+n_{B}+n_{C}+1\right)\left|\mu_{A}\right|^{2}\left|\mu_{B}\right|^{2}\left|\mu_{C}\right|^{2} .
\end{aligned}
$$


If the three qubits are in $\mathrm{W}$ state, their density matrix can be obtained as

$$
\begin{aligned}
\rho_{A B C}(W)= & C_{A}^{2} C_{B}^{2} C_{C}^{2} \sum_{n_{A}, n_{B}, n_{C}} \frac{e^{-2 \pi\left(n_{A} \Omega_{A} / a_{A}+n_{B} \Omega_{B} / a_{B}+n_{C} \Omega_{C} / a_{C}\right)}}{Z_{n_{A} n_{B} n_{C}}}[|001\rangle\langle 010| \\
& +|001\rangle\langle 100|+| 100\rangle\langle 001|+| 100\rangle\langle 010|+| 010\rangle\langle 001|+| 010\rangle\langle 100| \\
& +n_{B}\left|\mu_{B}\right|^{2}|011\rangle\left\langle\left. 110\left|+n_{A}\right| \mu_{A}\right|^{2} \mid 101\right\rangle\left\langle\left. 110\left|+n_{C}\right| \mu_{C}\right|^{2} \mid 011\right\rangle\langle 101| \\
& +n_{A}\left|\mu_{A}\right|^{2}|110\rangle\left\langle\left. 101\left|+n_{C}\right| \mu_{C}\right|^{2} \mid 101\right\rangle\left\langle\left. 011\left|+n_{B}\right| \mu_{B}\right|^{2} \mid 110\right\rangle\langle 011| \\
& +\left(\left(n_{A}+1\right)\left|\mu_{A}\right|^{2}+\left(n_{B}+1\right)\left|\mu_{B}\right|^{2}+\left(n_{C}+1\right)\left|\mu_{C}\right|^{2}\right)|000\rangle\langle 000| \\
& +\left(1+\left(n_{A}+1\right) n_{C}\left|\mu_{A}\right|^{2}\left|\mu_{C}\right|^{2}+\left(n_{B}+1\right) n_{C}\left|\mu_{B}\right|^{2}\left|\mu_{C}\right|^{2}\right)|001\rangle\langle 001| \\
& +\left(1+\left(n_{A}+1\right) n_{B}\left|\mu_{A}\right|^{2}\left|\mu_{B}\right|^{2}+n_{B}\left(n_{C}+1\right)\left|\mu_{B}\right|^{2}\left|\mu_{C}\right|^{2}\right)|010\rangle\langle 010| \\
& +\left(\left(n_{A}+1\right) n_{B} n_{C}\left|\mu_{A}\right|^{2}\left|\mu_{B}\right|^{2}\left|\mu_{C}\right|^{2}+n_{B}\left|\mu_{B}\right|^{2}+n_{C}\left|\mu_{C}\right|^{2}\right)|011\rangle\langle 011| \\
& +\left(1+n_{A}\left(n_{B}+1\right)\left|\mu_{A}\right|^{2}\left|\mu_{B}\right|^{2}+n_{A}\left(n_{C}+1\right)\left|\mu_{A}\right|^{2}\left|\mu_{C}\right|^{2}\right)|100\rangle\langle 100| \\
& +\left(n_{A}\left(n_{B}+1\right) n_{C}\left|\mu_{A}\right|^{2}\left|\mu_{B}\right|^{2}\left|\mu_{C}\right|^{2}+n_{A}\left|\mu_{A}\right|^{2}+n_{C}\left|\mu_{C}\right|^{2}\right)|101\rangle\langle 101| \\
& +\left(n_{A}\left|\mu_{A}\right|^{2}+n_{B}\left|\mu_{B}\right|^{2}+n_{A} n_{B}\left(n_{C}+1\right)\left|\mu_{A}\right|^{2}\left|\mu_{B}\right|^{2}\left|\mu_{C}\right|^{2}\right)|110\rangle\langle 110| \\
& \left.+\left(n_{A} n_{B}\left|\mu_{A}\right|^{2}\left|\mu_{B}\right|^{2}+n_{A} n_{C}\left|\mu_{A}\right|^{2}\left|\mu_{C}\right|^{2}+n_{B} n_{C}\left|\mu_{B}\right|^{2}\left|\mu_{C}\right|^{2}\right)|111\rangle\langle 111|\right],
\end{aligned}
$$

where

$$
\begin{aligned}
Z_{n_{A} n_{B} n_{C}}= & 3+\left(3 n_{A}+1\right)\left|\mu_{A}\right|^{2}+\left(3 n_{B}+1\right)\left|\mu_{B}\right|^{2}+\left(3 n_{C}+1\right)\left|\mu_{C}\right|^{2} \\
& +\left(3 n_{A} n_{B}+n_{A}+n_{B}\right)\left|\mu_{A}\right|^{2}\left|\mu_{B}\right|^{2}+\left(3 n_{B} n_{C}+n_{B}+n_{C}\right)\left|\mu_{B}\right|^{2}\left|\mu_{C}\right|^{2} \\
& +\left(3 n_{A} n_{C}+n_{A}+n_{C}\right)\left|\mu_{A}\right|^{2}\left|\mu_{C}\right|^{2} \\
& +\left(3 n_{A} n_{B} n_{C}+n_{A} n_{B}+n_{B} n_{C}+n_{A} n_{C}\right)\left|\mu_{A}\right|^{2}\left|\mu_{B}\right|^{2}\left|\mu_{C}\right|^{2} .
\end{aligned}
$$

The tripartite entanglement is the genuine three-party entanglement that cannot be reduced to any bipartite entanglement. We use the negativity three-tangle as the measure of the tripartite entanglement [35], which is defined as

$$
\pi \equiv \frac{1}{3}\left(\pi_{A}+\pi_{B}+\pi_{C}\right)
$$

where

$$
\pi_{A} \equiv \mathcal{N}_{A(B C)}^{2}-\mathcal{N}_{A B}^{2}-\mathcal{N}_{A C}^{2},
$$

with

$$
\begin{aligned}
\mathcal{N}_{A(B C)} & \equiv\left\|\rho_{A B C}^{T_{A}}\right\|-1, \\
\mathcal{N}_{A B} & \equiv\left\|\rho_{A B}^{T_{A}}\right\|-1 .
\end{aligned}
$$

Here $\rho_{A B C}$ is the density matrix of the three qubits, $\rho_{A B}$ is the reduced density matrix of $A$ and $B$, other quantities are similarly defined.

For simplicity, here we only present the result for the case that the accelerationfrequency ratios of the three qubits are the same. As shown in figure 8. For either GHZ or W state, the negativity three-tangle decreases with the increase of the acceleration-frequency ratio, and suddenly dies at a certain value. We have also found that entanglement sudden death generally occurs when at least two qubits accelerate, no matter whether the accelerations are equal. More details will be discussed elsewhere. 


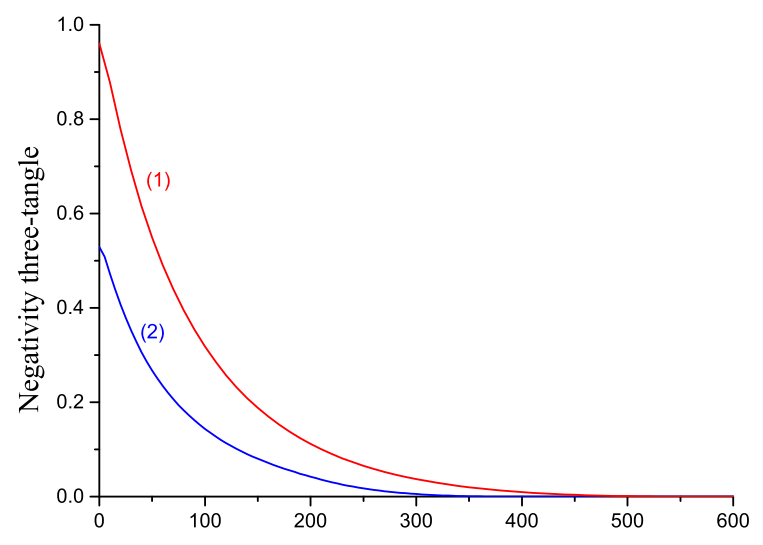

Figure 8. Negativity three-tangle as a function of the acceleration-frequency ratio of the three qubits in GHZ state (1) and in W state (2).

\section{Discussion and summary}

Now we come to the black hole. The spacetime outside its horizon is described by the Schwarzschild metric

$$
d s^{2}=\left(1-\frac{2 m}{r}\right) d t^{2}-\left(1-\frac{2 m}{r}\right)^{-1} d r^{2}-r^{2} d \theta^{2}-r^{2} \sin ^{2} \theta d \phi^{2}
$$

where the notations are standard. The proper acceleration of a static observer at $r$ is

$$
a=\frac{m}{r^{2}}\left(1-\frac{2 m}{r}\right)^{-1 / 2} \text {. }
$$

It is well known that near the horizon $r=2 m$, the Schwarzschild metric can be approximated as the Rindler metric $[10,36]$. The closer $r$ is to the horizon, the larger the acceleration. The uniform movement corresponds to free falling into the black hole.

Consider the entangled states studied above. Suppose at most one of them freely fall into the black hole, while the other is near the horizon $r=2 m$. According to the calculation above, we know that the entanglement between the qubits suddenly dies when one or more accelerating qubits are close enough to the horizon. For each qubit near the horizon, we have

$$
r \approx 2 m\left[1-\frac{1}{(4 m a)^{2}}\right]^{-1}
$$

from which the location of the entanglement sudden death can be determined.

Finally, one may wonder the reason of the entanglement sudden death as studied here. We think the Bosonic fields act as a drain of the entanglement originally exists between the qubits, because there are infinite number of Fock states $|n\rangle$ for each Bosonic field mode. In contrast, we conjecture that there is no entanglement sudden death if the fields are Fermionic as there are only two Fock states $|0\rangle$ and $|1\rangle$ for each Fermionic field mode. The absence of entanglement sudden death was recently noted in entanglement between Unruh modes [16]. Our work implicates that entanglement sudden death of the qubits can act 
as a probe of the nature of ambient fields coupled with the qubits and the existence of acceleration or gravity.

To summarize, we have studied the entanglement of two accelerating qubits coupled with scalar fields, and demonstrate the occurrence of its sudden death. We also found the entanglement sudden death of tripartite entanglement of three accelerating qubits in GHZ and $\mathrm{W}$ states. These results imply the entanglement sudden death of field-coupled qubits near the horizon of a black hole. This work might be useful to the issue of black hole firewall [37] or energetic curtain [38].

Open Access. This article is distributed under the terms of the Creative Commons Attribution License (CC-BY 4.0), which permits any use, distribution and reproduction in any medium, provided the original author(s) and source are credited.

\section{References}

[1] E. Schrödinger, Discussion of probability relations between separated systems, Proc. Cambr. Phil. Soc. 31 (1935) 555.

[2] S.A. Fulling, Nonuniqueness of canonical field quantization in Riemannian space-time, Phys. Rev. D 7 (1973) 2850 [INSPIRE].

[3] P.C.W. Davies, Scalar particle production in Schwarzschild and Rindler metrics, J. Phys. A 8 (1975) 609 [inSPIRE].

[4] B.S. DeWitt, Quantum field theory in curved space-time, Phys. Rept. 19 (1975) 295 [INSPIRE].

[5] W.G. Unruh, Notes on black hole evaporation, Phys. Rev. D 14 (1976) 870 [INSPIRE].

[6] W.G. Unruh and R.M. Wald, What happens when an accelerating observer detects a Rindler particle, Phys. Rev. D 29 (1984) 1047 [InSPIRE].

[7] L.C.B. Crispino, A. Higuchi and G.E.A. Matsas, The Unruh effect and its applications, Rev. Mod. Phys. 80 (2008) 787 [arXiv:0710.5373] [INSPIRE].

[8] V.F. Mukhanov, Introduction to quantum effects in gravity, Cambridge University Press, Cambridge U.K. (2007) [INSPIRE].

[9] I. Fuentes-Schuller and R.B. Mann, Alice falls into a black hole: entanglement in non-inertial frames, Phys. Rev. Lett. 95 (2005) 120404 [quant-ph/0410172] [INSPIRE].

[10] E. Martín-Martínez, L.J. Garay and J. León, Unveiling quantum entanglement degradation near a Schwarzschild black hole, Phys. Rev. D 82 (2010) 064006 [arXiv:1006.1394] [INSPIRE].

[11] M.-R. Hwang, D. Park and E. Jung, Tripartite entanglement in noninertial frame, Phys. Rev. A 83 (2001) 012111 [arXiv:1010.6154] [INSPIRE].

[12] M. Shamirzai, B.N. Esfahani and M. Soltani, Tripartite entanglements in non-inertial frames, Int. J. Theor. Phys. 51 (2012) 787 [arXiv:1103.0258] [INSPIRE].

[13] P.M. Alsing, I. Fuentes-Schuller, R.B. Mann and T.E. Tessier, Entanglement of Dirac fields in non-inertial frames, Phys. Rev. A 74 (2006) 032326 [quant-ph/0603269] [INSPIRE].

[14] Q. Pan and J. Jing, Degradation of non-maximal entanglement of scalar and Dirac fields in non-inertial frames, Phys. Rev. A 77 (2008) 024302 [arXiv:0802.1238] [INSPIRE]. 
[15] J. Wang and J. Jing, Multipartite entanglement of fermionic systems in noninertial frames, Phys. Rev. A 83 (2011) 022314 [arXiv: 1012.4268] [inSPIRE].

[16] B. Richter and Y. Omar, Degradation of entanglement between two accelerated parties: Bell states under the Unruh effect, Phys. Rev. A 92 (2015) 022334 [arXiv:1503.07526] [INSPIRE].

[17] Y. Shi, Entanglement in relativistic quantum field theory, Phys. Rev. D 70 (2004) 105001 [hep-th/0408062] [INSPIRE].

[18] T. Yu and J.H. Eberly, Sudden death of entanglement, Science 323 (2009) 598 [arXiv: 0910.1396].

[19] S.-Y. Lin, C.-H. Chou and B.L. Hu, Disentanglement of two harmonic oscillators in relativistic motion, Phys. Rev. D 78 (2008) 125025 [arXiv:0803.3995] [INSPIRE].

[20] B.L. Hu, S.-Y. Lin and J. Louko, Relativistic quantum information in detectors-field interactions, Class. Quant. Grav. 29 (2012) 224005 [arXiv: 1205.1328] [INSPIRE].

[21] R. Zhou, R.O. Behunin, S.Y. Lin and B.L. Hu, Boundary effects on quantum entanglement and its dynamics in a detector-field system, JHEP 08 (2013) 040 [arXiv:1301.0073] [INSPIRE].

[22] A.G.S. Landulfo and G.E.A. Matsas, Sudden death of entanglement and teleportation fidelity loss via the Unruh effect, Phys. Rev. A 80 (2009) 032315 [arXiv:0907. 0485] [INSPIRE].

[23] J. Doukas and L.C.L. Hollenberg, Loss of spin entanglement for accelerated electrons in electric and magnetic fields, Phys. Rev. A 79 (2009) 052109 [arXiv:0807.4356] [InSPIRE].

[24] J. Doukas and B. Carson, Entanglement of two qubits in a relativistic orbit, Phys. Rev. A 81 (2010) 062320 [arXiv:1003.2201] [InSPIRE].

[25] J. Hu and $\mathrm{H}$. Yu, Entanglement dynamics for uniformly accelerated two-level atoms, Phys. Rev. A 91 (2015) 012327 [arXiv:1501.03321] [INSPIRE].

[26] A. Datta, Quantum discord between relatively accelerated observers, Phys. Rev. A 80 (2009) 052304 [arXiv:0905.3301].

[27] L.C. Céleri, A.G.S. Landulfo, R.M. Serra and G.E.A. Matsas, Sudden change in quantum and classical correlations and the Unruh effect, Phys. Rev. A 81 (2010) 062130 [arXiv: 1003.4477] [INSPIRE].

[28] Y. Yao, X. Xiao, L. Ge, X.-g. Wang and C.-p. Sun, Quantum Fisher information in noninertial frames, Phys. Rev. A 89 (2014) 042336 [inSPIRE].

[29] J. Wang, Z. Tian, J. Jing and H. Fan, Quantum metrology and detection of Unruh effect, Sci. Rept. 4 (2014) 7195 [arXiv:1405.1940] [INSPIRE].

[30] P. Kok and U. Yurtsever, Gravitational decoherence, Phys. Rev. D 68 (2003) 085006 [gr-qc/0306084] [INSPIRE].

[31] G. Vidal and R.F. Werner, Computable measure of entanglement, Phys. Rev. A 65 (2002) 032314 [inSPIRE].

[32] M.B. Plenio, Logarithmic negativity: a full entanglement monotone that is not convex, Phys. Rev. Lett. 95 (2005) 090503 [quant-ph/0505071].

[33] W.K. Wootters, Entanglement of formation of an arbitrary state of two qubits, Phys. Rev. Lett. 80 (1998) 2245 [quant-ph/9709029] [INSPIRE]. 
[34] W. Dür, G. Vidal and J.I. Cirac, Three qubits can be entangled in two inequivalent ways, Phys. Rev. A 62 (2000) 062314 [INSPIRE].

[35] Y.C. Ou and H. Fan, Monogamy inequality in terms of negativity for three-qubit states, Phys. Rev. A 75 (2007) 062308.

[36] W. Rindler, Kruskal space and the uniformly accelerated frame, Am. J. Phys. 34 (1966) 1174 [INSPIRE].

[37] A. Almheiri, D. Marolf, J. Polchinski and J. Sully, Black holes: complementarity or firewalls?, JHEP 02 (2013) 062 [arXiv:1207.3123] [INSPIRE].

[38] S.L. Braunstein, S. Pirandola and K. Życzkowski, Better late than never: information retrieval from black holes, Phys. Rev. Lett. 110 (2013) 101301 [arXiv:0907.1190] [INSPIRE]. 